\title{
Engineering of Saccharomyces cerevisiae for anthranilate and methyl anthranilate production
}

\author{
Joosu Kuivanen ${ }^{1,2}$, Matti Kannisto ${ }^{1 *}$ (D) Dominik Mojzita ${ }^{1}$, Heiko Rischer ${ }^{1}$, Mervi Toivari ${ }^{1}$ and Jussi Jäntti ${ }^{1}$
}

\begin{abstract}
Background: Anthranilate is a platform chemical used by the industry in the synthesis of a broad range of high-value products, such as dyes, perfumes and pharmaceutical compounds. Currently anthranilate is produced via chemical synthesis from non-renewable resources. Biological synthesis would allow the use of renewable carbon sources and avoid accumulation of toxic by-products. Microorganisms produce anthranilate as an intermediate in the tryptophan biosynthetic pathway. Several prokaryotic microorganisms have been engineered to overproduce anthranilate but attempts to engineer eukaryotic microorganisms for anthranilate production are scarce.

Results: We subjected Saccharomyces cerevisiae, a widely used eukaryotic production host organism, to metabolic engineering for anthranilate production. A single gene knockout was sufficient to trigger anthranilate accumulation both in minimal and SCD media and the titer could be further improved by subsequent genomic alterations. The effects of the modifications on anthranilate production depended heavily on the growth medium used. By growing an engineered strain in SCD medium an anthranilate titer of $567.9 \mathrm{mg} \mathrm{I}^{\mathrm{I}^{-1}}$ was obtained, which is the highest reported with an eukaryotic microorganism. Furthermore, the anthranilate biosynthetic pathway was extended by expression of anthranilic acid methyltransferase 1 from Medicago truncatula. When cultivated in YPD medium, this pathway extension enabled production of the grape flavor compound methyl anthranilate in S. cerevisiae at $414 \mathrm{mg} \mathrm{I}^{-1}$.

Conclusions: In this study we have engineered metabolism of S. cerevisiae for improved anthranilate production. The resulting strains may serve as a basis for development of efficient production host organisms for anthranilate-derived compounds. In order to demonstrate suitability of the engineered S. cerevisiae strains for production of such compounds, we successfully extended the anthranilate biosynthesis pathway to synthesis of methyl anthranilate.
\end{abstract}

Keywords: Anthranilate, Methyl anthranilate, Saccharomyces cerevisiae, Metabolic engineering, Shikimate pathway

\section{Background}

Aromatic compounds varying from platform chemicals, such as polymer precursors, to high-value fine chemicals including flavour, fragrance and pharmaceutical substances are widely used in different industries. These compounds are typically derived from benzene, toluene and xylene obtained from petroleum or natural gas, or

\footnotetext{
*Correspondence: Matti.Kannisto@vtt.fi

${ }^{1}$ VTT Technical Research Centre of Finland Ltd, Espoo, Finland

Full list of author information is available at the end of the article
}

from secondary metabolites extracted from plant tissues. Recently, renewable production of aromatics and their derivatives using microbes has gained an increasing interest [1-4]. Among the compounds that are currently derived from petroleum, but could also be produced from renewables using industrial biotechnology, is the aromatic amine anthranilate (ANTH). ANTH is used for example as a precursor molecule in the production of dyes, fragrances and pharmaceuticals $[5,6]$. Its derivative methyl anthranilate (Me-ANTH), a naturally occurring 
plant-derived compound [7], constitutes a food flavour and cosmetic ingredient of significant demand.

ANTH is an intermediate in the microbial tryptophan (Trp) biosynthetic pathway and its production has been reported using engineered Escherichia coli [8], Pseudomonas putida [5] and Corynebacterium glutamicum [9] cells. In addition, production of a polymer precursor cis,cis-muconic acid (CCM) via ANTH in E. coli cells [10], and the flavor compound Me-ANTH in E. coli and C. glutamicum [9] have been reported.

Besides bacterial production hosts, the yeast Saccharomyces cerevisiae has raised interest as a chassis for aromatics production due to its robustness in bioprocess conditions [11] and the capability to express eukaryotic cytochrome P450 oxidases [12]-key enzymes in aromatic secondary metabolite pathways. S. cerevisiae has been engineered to convert ANTH to cinnamoyl, dihydrocinnamoyl and benzoyl anthranilates, potential pharmaceuticals [13]. These compounds were not however produced de novo, but ANTH feeding was required. Hitherto, ANTH production in S. cerevisiae had only been outlined in the patent literature [14]. Development and investigation of a $S$. cerevisiae platform for de novo ANTH production could provide a platform for ANTH and its many derivatives, and give useful insight of the less frequently engineered Trp pathway in S. cerevisiae.

Similarly to bacterial hosts, aromatic compounds in $S$. cerevisiae are mainly synthesized through the shikimate (SA) pathway and the following aromatic amino acid pathways for phenylalanine (Phe), tyrosine (Tyr) and Trp (Fig. 1a). SA pathway begins with the aldol reaction of erythrose-4-phosphate (E4P) and phosphoenolpyruvate (PEP) forming 3-deoxy-D-arabino-heptulosonate7-phosphate (DAHP), catalysed by the DAHP synthases (EC 2.5.1.54) Aro3 and Aro4. In the next five reactions, DAHP is converted to 5-enolpyruvyl-shikimate-3-phosphate (EPSP) via 3-dehydroquinate (DHQ), 3-dehydroshikimate (DHS), SA and shikimate-3-phosphate (S3P). In bacteria, these reactions are catalysed by separate enzymes but in S. cerevisiae, the conversion is carried out by the action of the pentafunctional enzyme Aro1 [15]. The last reaction from EPSP is catalysed by Aro2 resulting in formation of chorismate $(\mathrm{CHO})-\mathrm{a}$ common precursor of the aromatic amino acids. After $\mathrm{CHO}$ formation, separate branches diverge for the biosynthesis of Phe, Tyr and Trp. ANTH is the first intermediate in the Trp biosynthetic pathway and it is produced by Trp2/Trp3 complex in a reaction where an amide group

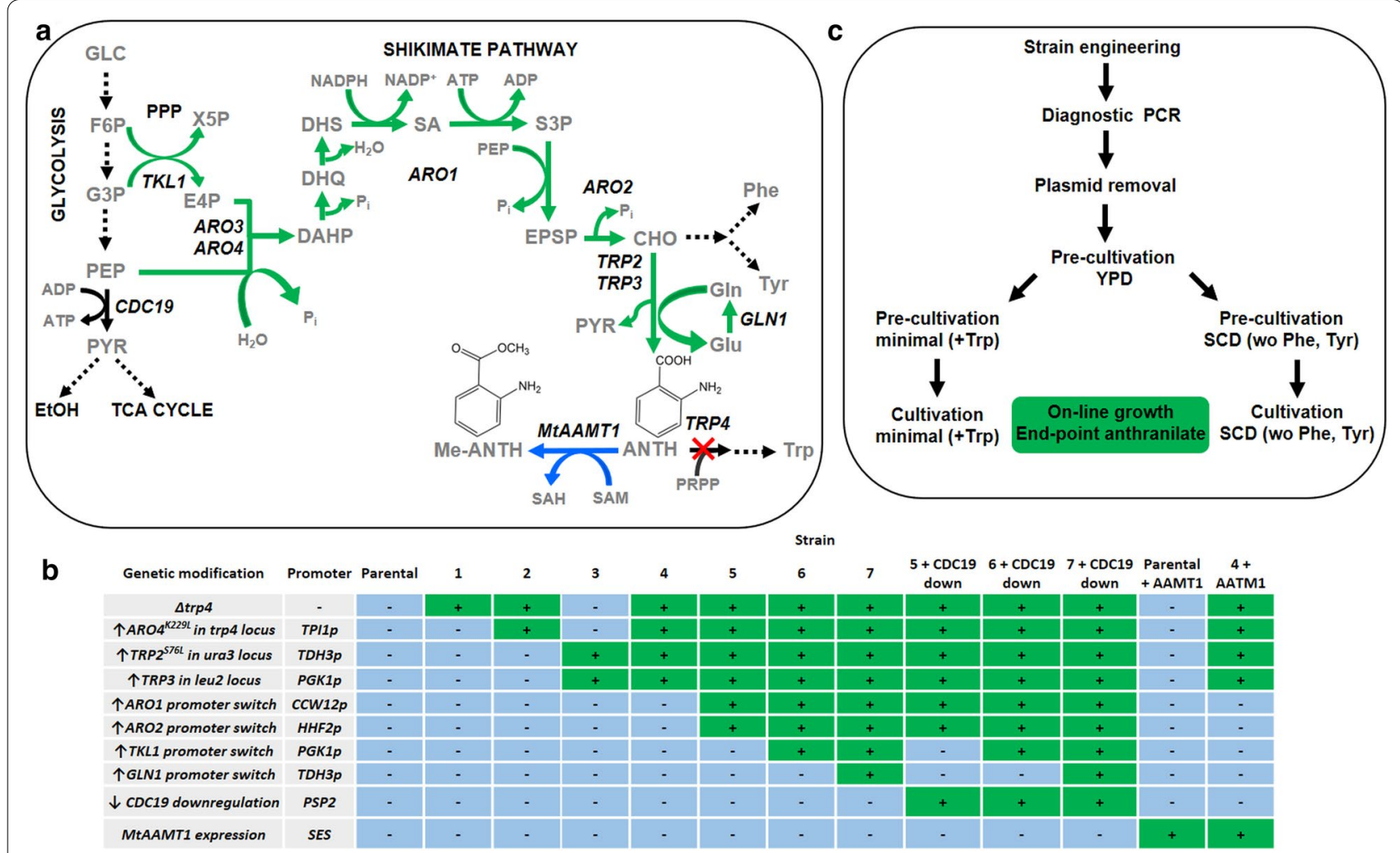

Fig. 1 a A simplified representation of the metabolic pathways engineered in this study. The upregulated reactions are shown with green arrows and the eliminated reaction is shown with a crossed arrow. The reaction added to extend the pathway to Me-ANTH synthesis is shown with a blue arrow. $\mathbf{b}$ Modifications done to the strains generated in this study. $\mathbf{c}$ Workflow of the construction and characterization of the strains 
is transferred from glutamine (Gln) to $\mathrm{CHO}$ resulting in formation of ANTH and glutamate (Glu). Gln synthase encoded by GLN1 regenerates the consumed Gln from Glu and ammonia. The SA pathway is regulated by the allosteric inhibition of several enzymes, such as, Aro3, Aro4 [4] and Trp2. In addition, the flux through the SA pathway is limited by the availability of PEP, which is converted to pyruvate by the main pyruvate kinase of S. cerevisiae, encoded by $C D C 19$ [16]. The availability of E4P from the pentose phosphate pathway (PPP) can also be considered a limiting factor because the carbon flux through E4P is low in S. cerevisiae [17].

In this study, the de novo production of ANTH is investigated in S. cerevisiae. Several upstream pathway genes were overexpressed by editing the chromosomal promoter regions of the target genes using a rapid CRISPR/Cas9 genome editing approach. In addition, the allosteric regulation of the SA pathway and the Trp branch was relieved with chromosomally integrated mutant enzymes. One of the ANTH overproduction strains was further engineered to produce the grape flavour compound, Me-ANTH, by expressing anthranilic acid methyltransferase 1 (MtAAMT1) from Medicago truncatula. To the best of our knowledge, besides the patent literature [14] describing ANTH production in $S$. cerevisiae, this is the first report on engineering an eukaryotic strain for ANTH or Me-ANTH production.

\section{Results}

\section{Construction and characterization of the strains}

We used a prototroph $S$. cerevisiae strain CEN.PK113$1 \mathrm{~A}$ as a platform for the engineering of the ANTHproducing S. cerevisiae strain. Gene deletions and the replacements of the native target gene promoters were generated using the two-plasmid CRISPR/Cas9 system described previously [18]. The plasmid expressing an sgRNA was transformed as PCR amplified fragments into a target $S$. cerevisiae strain that already maintained the Cas9 expression plasmid (see Additional file 1: Fig. S1), a method shown to enable a quick turnaround time in each engineering cycle [19]. The strains (Fig. 1b) were cultivated in microtiter plates using a cultivation robot [18], and both the optical density (OD) values together with the ANTH production were measured at the end of cultivation. Two different cultivation media were tested-the SCD medium containing $2 \%$ glucose and amino acids (except Phe and Tyr) and the Verduyn minimal medium containing $1 \%$ glucose and supplemented by $\operatorname{Trp}$ (due to the auxotrophy after the absence of Trp4 activity). The modified metabolic steps, generated strain and workflow are presented in Fig. 1a-c, respectively.

\section{Triggering anthranilate accumulation by disruption of TRP4}

The wild type strain did not produce ANTH in either media. When the TRP4 gene, encoding an ANTH phosphoribosyl transferase, was disrupted (strain 1), ANTH titers of 11.4 and $42.9 \mathrm{mg} \mathrm{l}^{-1}$ were detected in SCD and minimal medium, respectively (Fig. 2a, c). A slight growth defect caused by TRP4-deletion was observed in SCD but not in minimal medium (Fig. 2b, d).

\section{Relieving allosteric regulation of Aro4 and Trp2 and overexpression of TRP3}

To overcome feedback-inhibition of the DAHP synthases, we introduced the feedback insensitive variant $A R O 4^{K 229 L}$ [20] of a DAHP synthase as a single-copy genomic integration into the trp4 locus without removing the native ARO4 (strain 2). This modification improved ANTH titer in SCD medium to $88.4 \mathrm{mg} \mathrm{l}^{-1}$ and in minimal medium to $104.5 \mathrm{mg} \mathrm{l}^{-1}$, but caused a small growth defect in SCD medium (Fig. 2b).

A feedback insensitive mutant $T R P 2^{S 76 L}$ [21] was introduced together with TRP3 overexpression cassette as single-copy genomic integrations into the ura 3 and leu2 loci, respectively, of the parental strain (to form strain 3) and the $\triangle T R P 4-A R O 4^{K 229 L}$ strain (to form strain 4) (Fig. 1b). Interestingly, the strain 3 without the deletion of TRP4 nor the expression of $A R O 4^{K 229 L}$ produced similar titers of ANTH and had a more severe growth defect compared to strain 2 . It is unlikely for the growth defects in the ANTH-producing strains described here to be due to toxicity of ANTH since it has been shown that yeast growth is not inhibited even at an ANTH concentration of $2 \mathrm{~g} \mathrm{l}^{-1}$ [22]. When both feedback insensitive enzymes Aro4 ${ }^{\mathrm{K} 229 \mathrm{~L}}$ and $\operatorname{Trp} 2^{\mathrm{S} 76 \mathrm{~L}}$ were combined (strain 4), the observed ANTH titers were doubled in both SCD (209.9 $\mathrm{mg} \mathrm{l}^{-1}$ ) and minimal (199.7 $\mathrm{mg} \mathrm{l}^{-1}$ ) media. Also the growth reduced significantly compared to the previous strains $1-3$ (Fig. 2b, d).

\section{Activation of $A R O 1, A R O 2$ and $T K L 1$ by promoter replacements}

Next, the SA pathway genes $A R O 1$ and $A R O 2$ were overexpressed by replacing the native promoters with known strong constitutive yeast promoters (Fig. 1b). The replacement was implemented through duplexed CRISPR genome edits into strain 4, forming strain 5 . In SCD medium, the observed ANTH titer increased from $209.9 \mathrm{mg} \mathrm{l}^{-1}$ (strain 4) up to $367.9 \mathrm{mg} \mathrm{l}^{-1}$ while the growth remained at the same level (Fig. 2a, b). In contrast, a severe growth defect was observed in minimal medium resulting in low ANTH titer (Fig. 2c, d). However, when ANTH titer was normalized to OD (Fig. 2e, 

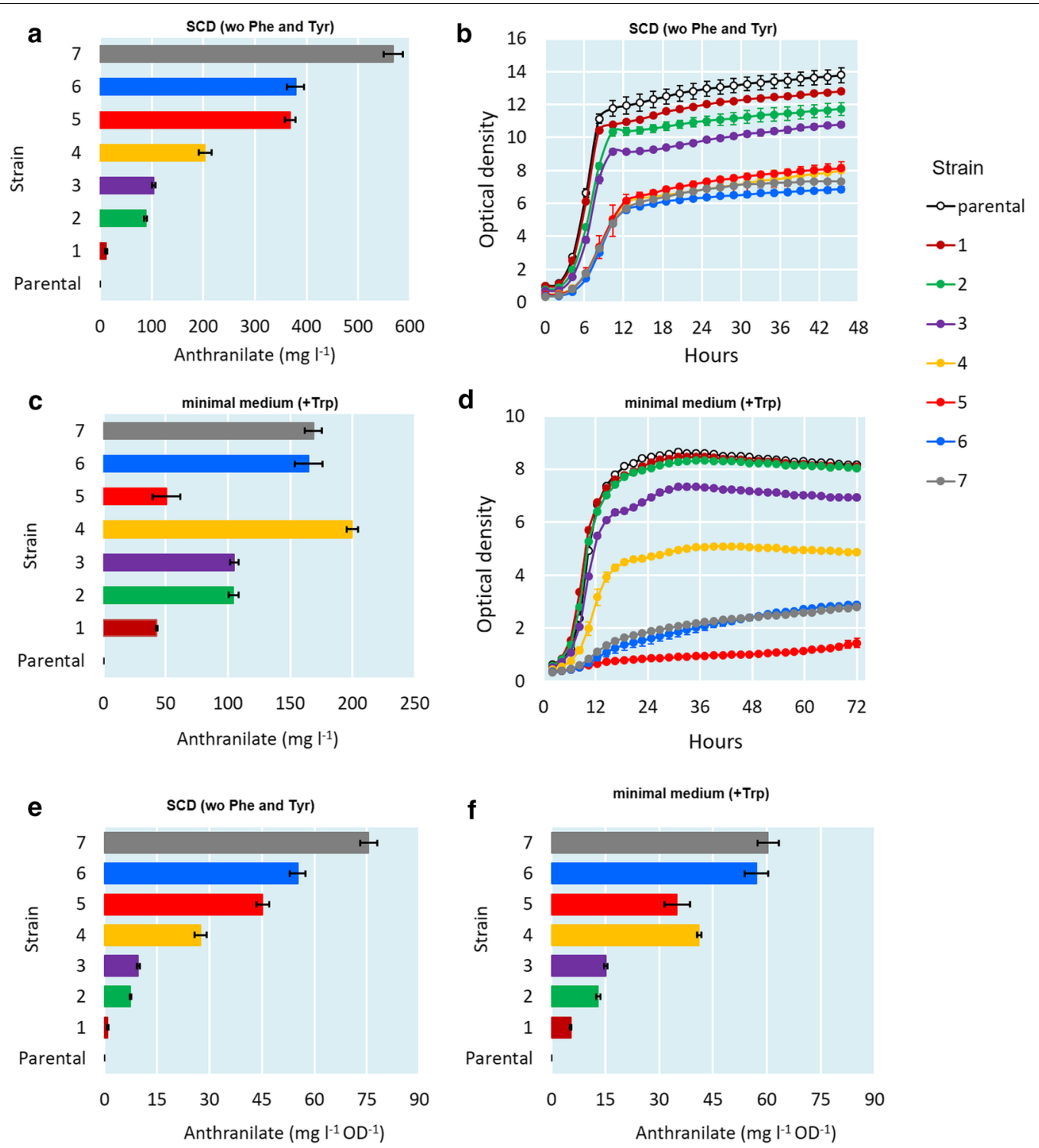

Fig. 2 ANTH titers at the end-point of the cultivations of the engineered strains 1-7 in SCD (a) and minimal medium (c). ODs of the cultivations in SCD (b) and minimal medium (d). The end-point ANTH titers divided by the end-point ODs of the engineered strains 1-7 in SCD (e) and minimal medium (f)

f), the production in minimal medium by the strain 5 (35.0 $\left.\mathrm{mg} \mathrm{l}^{-1} \mathrm{OD}^{-1}\right)$ remained at a higher level than with the strains $1-3\left(5.3-15.2 \mathrm{mg} \mathrm{l}^{-1} \mathrm{OD}^{-1}\right)$, but still lower as compared to the strain $4\left(41.1 \mathrm{mg} \mathrm{l}^{-1} \mathrm{OD}^{-1}\right)$.

Overexpression of the transketolase TKL1 potentially increasing flux towards E4P into strain 5 (resulting in strain 6) had a slightly negative effect on growth as compared to strain 5 , but no significant effect on ANTH titer in SCD (Fig. 2a, b). However, OD normalized ANTH production was increased from $45.2 \mathrm{mg} \mathrm{l}^{-1}$ $\mathrm{OD}^{-1}$ to $55.2 \mathrm{mg} \mathrm{l}^{-1} \mathrm{OD}^{-1}$ (Fig. 2e). In minimal medium, the growth defect resulting from ARO1 and ARO2 overexpression in strain 5 was partially restored (Fig. 2d). In addition, the OD normalized ANTH production was higher $\left(57.1 \mathrm{mg} \mathrm{l}^{-1} \mathrm{OD}^{-1}\right)$ compared to any of the previous strains 1-5 (5.3-41.1 $\left.\mathrm{mg} \mathrm{l}^{-1} \mathrm{OD}^{-1}\right)$ (Fig. 2f).

\section{Overexpression of the Gln synthase GLN1}

To ensure sufficient Gln supply, we activated the native GLN1 expression by replacing its native promoter with a strong constitutive yeast promoter (Fig. 1b). As a result, when grown in SCD medium, we observed a significant increase in the ANTH titer from $378.8 \mathrm{mg} \mathrm{l}^{-1}$ (strain 6) to $567.9 \mathrm{mg} \mathrm{l}^{-1}$ (strain 7) (Fig. 2a). At the same 
time, only a slight reduction on growth was observed (Fig. 2b). However, the benefit from GLN1 activation was not observed when the strain was grown in minimal medium (Fig. 2c, d, f).

\section{Down-regulating the pyruvate kinase $C D C 19$}

We further attempted to increase the PEP flux towards ANTH by regulating the CDC19 expression in the strains 5, 6 and 7. Attempts to generate a glucose-growing null mutant failed, but we succeeded in replacing the native promoter with the PSP2 promoter that has been shown to be a weak promoter [23]. In SCD medium, the OD normalized ANTH production improved slightly in the modified strain 7 (from 75.5 to $92.4 \mathrm{mg} \mathrm{l}^{-1} \mathrm{OD}^{-1}$ ) but not in the modified strains 5 (from 45.2 to $32.8 \mathrm{mg} \mathrm{l}^{-1} \mathrm{OD}^{-1}$ ) and 6 (from 55.2 to $53.6 \mathrm{mg} \mathrm{l}^{-1} \mathrm{OD}^{-1}$ ) (Fig. 3a). A clear growth defect was observed in the modified strains 6 and 7 , but not in the strain 5 . In contrast, only the strain 5 showed an increase in the ANTH titer (from 35.0 to $53.5 \mathrm{mg} \mathrm{l}^{-1} \mathrm{OD}^{-1}$ ) in minimal medium (Fig. 3c). However, the growth of the strain 5 without the CDC19 down-regulation (Fig. 3d) was very poor, which may affect this observation. Interestingly, the $C D C 19$ downregulation restored the growth of strain 5 significantly. With the strains 6 and 7 in minimal medium, the downregulation affected the growth negatively.

\section{Production of methyl anthranilate by expression of the MtAAMT1 gene}

The strain 4 was selected a base strain for the production of Me-ANTH due to its relatively fast growth and yet decent production of ANTH as compared to more engineered strains observed in minimal medium (Fig. 2c, d). The MtAAMT1 gene, encoding the M. truncatula anthranilic acid methyltransferase 1 , was introduced as a single-copy genomic integration into the strain 4 , and in the parental strain. The MtAAMT1 gene was expressed using the synthetic expression system (SES) [24] to achieve constitutive high production of the enzyme (see Additional file 1: Fig. S2). The identity of the products were confirmed by UPLC-MS analysis (see Additional file 1: Fig. S3). The modified strain 4 produced Me-ANTH to amounts similar to the amounts of ANTH produced by the original stain 4 (Fig. 4), indicating virtually complete conversion of ANTH into Me-ANTH by the introduced methyltransferase. The titer of Me-ANTH exceeded $400 \mathrm{mg} \mathrm{l}^{-1}$ in YPD medium at day 5 .

In order to verify the suitability of the strain 4 as a chassis and YPD as a medium for Me-ANTH production, strains 4 and 7 were cultivated in YPD medium and strain 4, with or without MtAAMT1 expression, in SCD medium or minimal medium for 5 days. OD values were measured and ANTH and Me-ANTH titers determined from samples taken at the end point of the cultivation (Fig. 5). Strain 4 grew to a higher OD

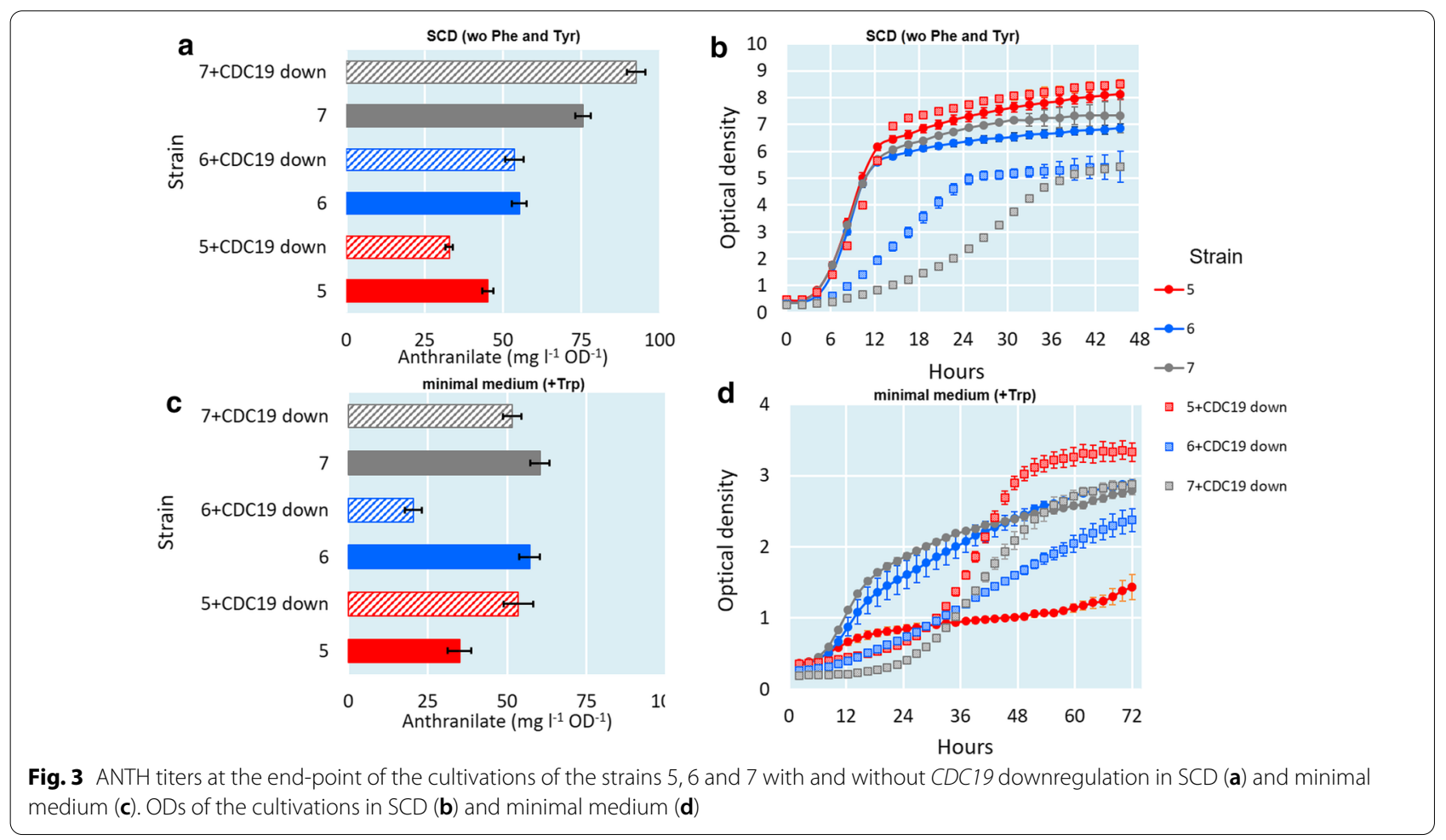



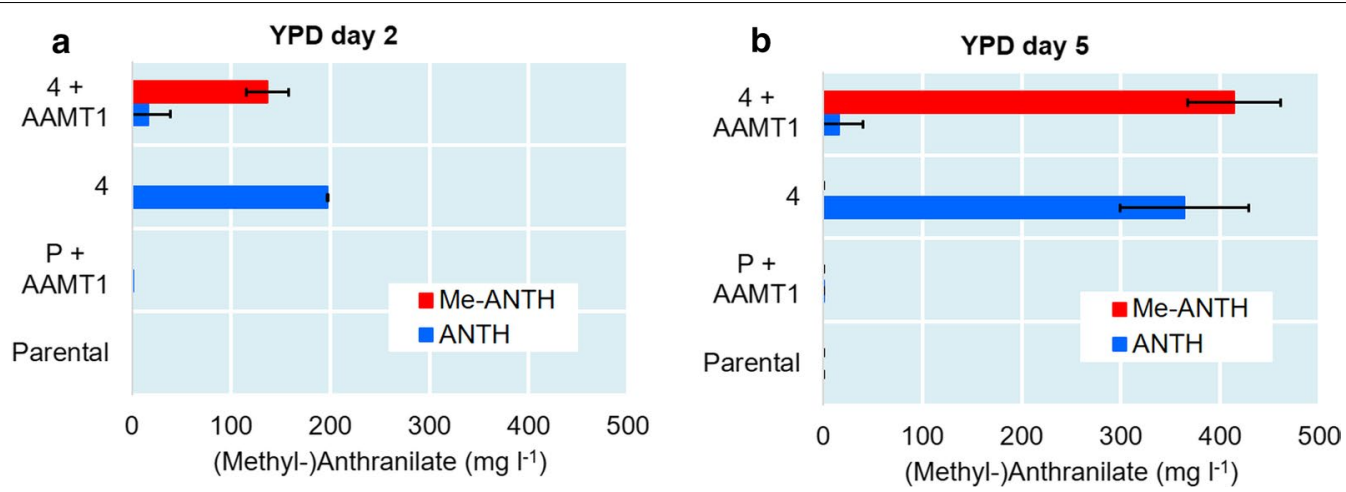

Fig. 4 ANTH and Me-ANTH titers in the cultivations of the parental strain and the strain 4, with and without the MtAAMT1 expression cassette. The cultivations were performed in YPD medium and the products analysed in the culture supernatants at day 2 (a) and day 5 (b)
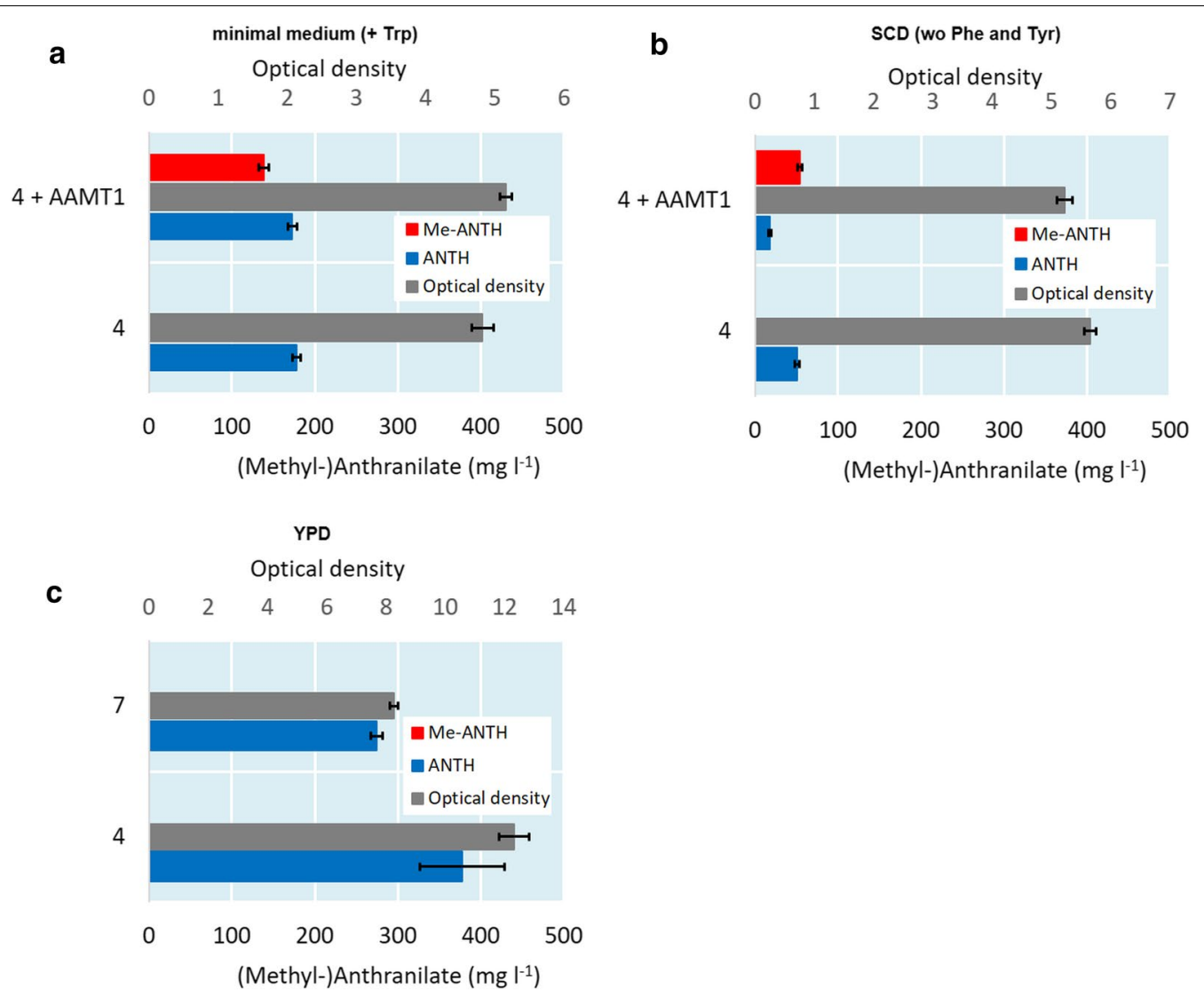


\section{Discussion}

ANTH overproduction has been engineered in several prokaryotes but the suitability of eukaryotic species for ANTH production has received little attention. Only the patent application by Jäger et al. [14] suggest that ANTH production can be triggered in S. cerevisiae by disruption of TRP4. We attempted to engineer an efficient ANTH producing $S$. cerevisiae by first disrupting TRP4 from the genome of S. cerevisiae CEN.PK113-1A and then carrying out extensive modifications to its genome to improve carbon flux towards ANTH biosynthesis. We introduced $A R O 4^{K 229 L}$, a gene for Aro4 mutant insensitive to feedback inhibition by Tyr [25], to the TRP4 locus. ARO4 ${ }^{K 229 L}$ has previously been successfully used in S. cerevisiae for example to improve the production of CCM [26], and double the product yield without affecting growth in a $S$. cerevisiae strain engineered to produce coumarate [27]. Here, the use of $A R O 4^{K 229 L}$ improved ANTH titer in both media tested but did not affect growth in minimal medium and only very slightly in SCD medium.

We overexpressed TRP2 ${ }^{S 76 L}$ and TRP3 to funnel carbon towards ANTH production and reduce the flux to Phe and Tyr biosynthesis. This modification reduced OD slightly but did not affect ANTH titers significantly, thus increasing the OD normalized ANTH production. In the reaction catalyzed by Trp2 and Trp3 a pyruvate molecule is also formed. This pyruvate-forming reaction has been used to couple ANTH production to growth in E. coli by knocking out all other metabolic reactions producing pyruvate [28].

It has been shown that the overexpression of $A R O 1$ and ARO2 improves Tyr production in rich medium in a metabolically engineered $S$. cerevisiae strain [29]. This strain, which had also TKL1 expression upregulated, had also a slight growth defect [29]. In the experiments of Gold et al. [27], overexpression of ARO1 in a S. cerevisiae strain deleted for ARO10 knockout and expressing the $A R O 4^{K 229 L}$ mutant improved production of the heterologous product coumarate without a significant effects on growth in the minimal medium. We show here that the activation of $A R O 1$ and $A R O 2$ improves ANTH production and reduces growth of the engineered $S$. cerevisiae in minimal but not in SCD medium (strain $4 \rightarrow 5$ ). The $p$-hydroxyphenylpyruvate, an intermediate of Tyr biosynthesis, acts as an inhibitor to $S$. cerevisiae transketolase [30]. Thus, the overexpression of $A R O 1$ and $A R O 2$, which increase flux towards Tyr biosynthesis, might have reduced transketolase activity. This would explain why overexpression of TKL1 (strain $5 \rightarrow 6$ ) restored growth slightly in minimal medium. As a growth defect was not seen in the amino acid containing SCD medium, it is likely that the reduction in growth in the minimal medium was due to reduced synthesis of some other metabolites, such as E4P. Furthermore, downregulation of the gene for pyruvate kinase, $C D C 19$, drastically improved the growth of the strain 5 . Decrease in pyruvate kinase activity can be expected to result in the accumulation of three carbon intermediates of glycolysis, including G3P which is converted to E4P and X5P in a reaction that consumes also one molecule of F6P. Thus, it seems likely that the overexpression of $A R O 1$ and $A R O 2$ resulted in a growth defect through the depletion of the E4P pool, either directly by funnelling E4P towards SA pathway or by $p$-hydroxyphenylpyruvate-mediated transketolase inhibition. Deletion of glucose-6-phosphate dehydrogenase, $Z W F 1$, and activation of transketolase, $T K L 1$, have been shown to improve the product yield of the SA pathway derived CCM [26]. However, deletion of $Z W F$ decreases the NADPH pool and if the product synthesis requires NADPH, the $Z W F 1$ deletion may have a negative effect as shown previously [31]. For this reason, we only activated the TKL1 but did not delete $Z W F 1$ (strain $5 \rightarrow 6$ ).

Expression of Gln synthase $(g \ln A)$ has been shown to increase ANTH yield in a minimal medium of a metabolically engineered E. coli strain [10]. Glu has been also shown to accumulate as a major side-product in an E. coli strain engineered for Trp production [32]. We upregulated GLN1 expression in the engineered S. cerevisiae but did not observe improved ANTH titer in minimal medium while in SCD medium the ANTH titer improved significantly. It could be that either concentration of Gln is not limiting ANTH production or that the substrate of Gln synthase, Glu, is not present at sufficiently high concentration to affect ANTH production when strain 6 is cultivated in minimal medium.

Down-regulation of pyruvate kinase reaction has been considered as a potential approach to redirect the carbon flux towards aromatic amino acid biosynthesis. However, introduction of the mutation T21E in the CDC19 gene, which is known to reduce pyruvate kinase activity, has been shown not to increase flux of carbon to the aromatic amino acid biosynthesis pathway [27]. As pointed out by Suástegui et al. [31], this experiment was carried out in a ZWF1 knockout strain which might have affected the results. Since it has been shown that the $C D C 19$ null mutant cannot grow on glucose [33], we attempted to increase availability of PEP to the SA pathway in the strains $5-7$ by downregulating pyruvate kinase activity. Growth was negatively affected in both SCD and minimal media for strains 6 and 7. In contrast, growth of strain 5 was greatly improved in minimal medium. ANTH titer improved for strain 7 in SCD medium and for strain 5 in minimal medium, while in other cases the ANTH titers either decreased or remained at the same level. Therefore, it seems that the effects of pyruvate kinase 
down-regulation are highly dependent on the genetic background of the strain.

ANTH production has been successfully engineered in several prokaryotic microorganisms. For example, $P$. putida has been engineered to produce a titer of $0.25 \mathrm{~g} \mathrm{l}^{-1}$ of ANTH in a shake flask cultivation and a titer of $1.54 \mathrm{~g} \mathrm{l}^{-1}$ and a yield of $0.035 \mathrm{~g}_{\text {ANTH }} \mathrm{g}_{\text {glucose }}{ }^{-1}$ in a fed-batch cultivation in a minimal medium [5]. E. coli engineered for ANTH production produced a titer of $0.75 \mathrm{~g} \mathrm{l}^{-1}$ and a yield of $0.20 \mathrm{~g}_{\text {ANTH }} \mathrm{g}_{\text {glucose }}{ }^{-1}$ in a batch cultivation in a minimal medium and a titer of $14 \mathrm{~g} \mathrm{l}^{-1}$ and a yield of $0.20 \mathrm{~g}_{\text {ANTH }} \mathrm{g}_{\text {glucose }}{ }^{-1}$ in a fed-batch cultivation in rich medium [8]. E. coli and C. glutamicum have also been engineered for Me-ANTH production [9]. By fed-batch cultivating these strains in minimal medium, a Me-ANTH titer of $4.47 \mathrm{~g} \mathrm{l}^{-1}$ and a yield of 0.045 $\mathrm{g}_{\text {Me-ANTH }} \mathrm{g}_{\text {glucose }}{ }^{-1}$ were obtained with $E$. coli and a titer of $5.74 \mathrm{~g} \mathrm{l}^{-1}$ and a yield of $0.020 \mathrm{~g}_{\mathrm{Me}-\mathrm{ANTH}} \mathrm{g}_{\text {glucose }}{ }^{-1}$ were obtained with C. glutamicum. Unlike prokaryotic host organisms, such as E. coli and $P$. putida which lack internal membranes, $S$. cerevisiae can express cytochrome P450 oxidases of eukaryotic origin [12], allowing production of a broader range of ANTH derived metabolites. Furthermore, ANTH production in S. cerevisiae is uncoupled from growth [14], allowing larger portion of the carbon source to be used in ANTH production. Currently the only reported attempt to engineer an eukaryote to produce ANTH can be found in the patent application by Jäger et al. who claim to have produced $320 \mathrm{mg} \mathrm{l}^{-1}$ of ANTH using an S. cerevisiae TRP4 knockout strain [14].

\section{Conclusions}

Here, we first knocked out the TRP4 gene from the genome of $S$. cerevisiae and then further engineered its metabolism for improved ANTH production. By carrying out batch cultivations with a strain optimized for ANTH production, we could obtain a titer of $199.7 \mathrm{mg} \mathrm{l}^{-1}$ in a minimal medium and a titer of $567.9 \mathrm{mg} \mathrm{l}^{-1}$ in SCD medium. Thus, we were able to nearly double the previously highest ANTH titer obtained with an eukaryotic microorganism. As these titers were obtained by cultivating the engineered $S$. cerevisiae strain on a microplate, drastically higher titers could be obtainable by controlled bioreactor cultivations e.g. in fed-batch mode. Furthermore, we show that these engineered $S$. cerevisiae strains are suitable for production of high value ANTH-derived compounds. By extending the pathway with a recently described anthranilic acid methyl transferase MtAAMT1 from $M$. truncatula [34] the industrially valuable flavor compound Me-ANTH accumulated. While the ANTH titers still remain relatively low compared to the values obtained with prokaryotes, we foresee that the strains presented here can serve as a basis for the development of efficient host organisms for the production of many ANTH-derived compounds.

\section{Methods}

Strains

All the plasmids were produced in E. coli TOP10 cells. The S. cerevisiae strains CEN.PK113-1A (MATa URA3 HIS3 LEU2 TRP1 MAL2-8c SUC2) and CEN.PK113-17A (MATa ura3-52 HIS3 leu2-3,112 TRP1 MAL2-8c SUC2) were used as parental strains. Detailed description of the engineered S. cerevisiae strains used in this study is shown in Fig. 1b. The strains used in the experiments where the Me-ANTH production was tested (shown in Figs. 4,5$)$ were constructed as follows. The parental strain and the strain 4 were transformed with the MtAAMT1 expression cassette released from the pHIS3in-kanMX SES-MtAAMT1 plasmid by NotI. The corresponding negative control strains were also constructed by transformations of the $\operatorname{kanMX}$ selection marker cassette released from the plasmid pHIS3in-kanMX by NotI. The transformed strains were selected for growth in presence of G-418 (YPD agar with $200 \mathrm{mg} \mathrm{l}^{-1}$ of G-418), and tested for the correct genomic integrations into the HIS3 locus by qPCR (primers listed in Table 1).

\section{Media and culture conditions}

Luria Bertani Broth culture medium supplemented with $100 \mu \mathrm{g} \mathrm{ml}^{-1}$ of ampicillin or $25 \mu \mathrm{g} \mathrm{ml}^{-1}$ of chloramphenicol and culture conditions of $37^{\circ} \mathrm{C}$ and $250 \mathrm{rpm}$ were used with $E$. coli. YPD medium $\left(10 \mathrm{~g} \mathrm{l}^{-1}\right.$ yeast extract, $20 \mathrm{gl}^{-1}$ peptone and $20 \mathrm{~g} \mathrm{l}^{-1} \mathrm{D}$-glucose) with $200 \mathrm{\mu g} \mathrm{ml}^{-1}$ of G418 was used for S. cerevisiae pre-cultures. For selection of transformants with TRP2 ${ }^{S 76 L}$ and TRP3 integration cassettes, SCD-Ura-Leu (uracil and leucine deficient synthetic complete media supplemented with $20 \mathrm{~g}$ D-glucose $1^{-1}$ ) plates were used for uracil auxotrophic selection. All the S. cerevisiae cultivations were carried out at $30{ }^{\circ} \mathrm{C}$ and small scale liquid cultivations were shaken at $250 \mathrm{rpm}$.

The characterization of the engineered $S$. cerevisiae strains was carried out in 96-well plates using a Beckman Coulter screening and cultivation robot as described previously [18]. Briefly, two sequential 24-h pre-cultivation steps in YPD and SCD-Phe-Tyr (Phe and Tyr deficient synthetic complete media supplemented with $20 \mathrm{~g} \mathrm{l}^{-1}$ D-glucose) or defined minimal medium [35] (supplemented with $0.4 \mathrm{mM}$ Trp and $10 \mathrm{~g} \mathrm{l}^{-1} \mathrm{D}$-glucose), respectively, were carried out (Fig. 1c). The final cultivations were done in SCD-Phe-Tyr medium for $45 \mathrm{~h}$ and minimal medium for $72 \mathrm{~h}$. The cultivation volume of $150 \mu \mathrm{l}$ was used in each step and inoculation volume of $10 \mu \mathrm{l}$ in each transfer. Cultivations were monitored by measuring $\mathrm{OD}$ at $595 \mathrm{~nm}$ in 2-h intervals. The strains were further 
Table 1 Primers used in the construction of the strains

\begin{tabular}{|c|c|}
\hline Primer & Sequence $\left(5^{\prime} \rightarrow 3^{\prime}\right)$ \\
\hline OJKLiF-016 & TGCTTAAATCTATAACTACAAAAAACACATACAGGAATTCCACCATGAGTGAATCTCCAATGTT \\
\hline OJKLiF-017 & CGTTACCCTTAGTAGTGGTGATAGCAGCAACACCATGCAAAGTAACACCCATGAAATGGTG \\
\hline OJKLiF-018 & AGCCGCTGCTCATTCTCACCATTTCATGGGTGTTACTTTGCATGGTGTTGCTGCTATCAC \\
\hline OJKLiF-019 & CTTATTCAGTTAGCTAGCTGAGCTCGACTCTAGAGGATCCCTATTTCTTGTTAACTTCTC \\
\hline OJKLiF-084 & ACTGACAACTAAGTGAATTTAAACTGCAATAATCACAAGAAAACTTAGTATTCCCTTATCCTAGTGTTTAAAGATTACGG \\
\hline OJKLiF-085 & TTCAGCTTAGTACAAATAAAACGTCTCTATCTAGTTTAAGTATAACATATGAAATAATGTCGAATTGGAGCTAGACAAAG \\
\hline OJKLiF-133 & GCATCGTCTCATCGGTCTCATATGACCGCTTCCATCAAAAT \\
\hline OJKLiF-134 & GTGGCGAGATACCTATGAATAAATAACGATCTAATTCATTA \\
\hline OJKLiF-135 & TATTCATAGGTATCTCGCCAC \\
\hline OJKLiF-136 & ATGCCGTCTCAGGTCTCAGGATTTAAGCTGATCCTACGATAT \\
\hline OJKLiF-137 & GCATCGTCTCATCGGTCTCATATGTCTGTGCACGCTGCAAC \\
\hline OJKLiF-138 & ATGCCGTCTCAGGTCTCAGGATTTATTCGCATAATTCATGAATG \\
\hline oNNF-003 & GACACCCGACAGATCAAGGC \\
\hline oNNF-004 & GCACGGTTATCCACAGAATC \\
\hline 8BS_mCh_GA_F & GATACTAACGCCGCCATCCAGTGTCGGCGCGCCGAATTAACCCTCACTAAAGG \\
\hline 8BS_mCh_GA_R & GATTCCATTGTAGATATTTAATTATGTGTGTTTATTCGAAACTAAGTTCTTG \\
\hline sTF_noPacl_GA_F & CGAATAAACACACATAATTAAATATCTACAATGGAATCTACTCCTACTAAGCAAAAAGC \\
\hline sTF_noPacl_GA_R & GCTGCAGCTTTAAATAATCGGTGTCAGATATCAGGATCAATGGCGTCGGACA \\
\hline MtAAMT1_GA_F & CTGCTTATCAACACACAAACACTTAATTAAAATGGAAGTTGCTCAAGTTTTACCAATG \\
\hline MtAAMT1_GA_R & CATAAATCATAAGAAATTCGGATCCTCAAGCTTTTCTAGTCATCATTAAAGTCAAATTAG \\
\hline MtAAMT1_qPCR_F & GAAATGGAAACTGAAATGGGTC \\
\hline MtAAMT1_qPCR_R & CGTTAGATGGTGATGTAGAAGTC \\
\hline SC_HIS3_qPCR_F & TCGCAAGTGATTAACGTCCA \\
\hline SC_HIS3_qPCR_R & CGCAAATCCTGATCCAAACCT \\
\hline kanMX_qPCR_F & TATTGTTGATGCGCTGGCAG \\
\hline kanMX_qPCR_R & TATTCATTCGTGATTGCGCC \\
\hline
\end{tabular}

characterized by cultivating 5 days in $50 \mathrm{ml}$ of SCDPhe-Tyr medium or minimal medium (strain 4) or YPD medium (strains 4 and 7). OD was measured and UPLCMS samples taken at the end point of the cultivation.

The production of Me-ANTH was performed in $50 \mathrm{ml}$ of SCD-Phe-Tyr medium, minimal medium or YPD medium. The strains were cultivated in duplicate (YPD medium) or triplicate (SCD-Phe-Tyr medium and minimal medium) liquid cultures in $250 \mathrm{ml}$ Erlenmeyer flasks for 5 days at $30{ }^{\circ} \mathrm{C}$ with a shaking of $220 \mathrm{rpm}$. The culture supernatant samples were collected by centrifugation in day 2 and day 5 , and subjected to the UPLC-MS analysis.

Unless stated otherwise, all cultivations were carried out in triplicates. Figures 2, 3, 4, 5 represent average values from measurements taken from these cultivations and error bars represent standard deviations.

\section{Plasmids and DNA construction}

Expression cassette for the allosterically insensitive DAHP synthase $A R O 4^{K 229 L}$ was constructed by PCR amplifying the $A R O 4^{K 229 L}$ open reading frame (ORF) in two parts from $S$. cerevisiae genomic DNA with the primers oJKLiF-016/-017 and oJKLiF-018/-019 (Table 1). The primers oJKLiF-017 and -018 introduced the $K 229 \mathrm{~L}$ mutation and contained the homology arms for mutual recombination. Primers oJKLiF-016 and -019 contained the homology arms for the EcoRI and BamHI digested yeast expression plasmid derived from pYX212 [36]. These two PCR fragments were combined and cloned into the digested expression plasmid backbone using the yeast homologous recombination. As a result, $A R O 4^{K 229 L}$ was in the yeast expression plasmid under TPI1p. For genomic integrations, $A R O 4^{K 229}$ expression cassette was PCR amplified with the primers oJKLiF-084/-085, introducing homology arms for the genomic TRP4 integration, respectively. Similar to $A R O 4^{K 229 L}$, allosterically insensitive TRP2 $2^{S 76 L}$ mutant was PCR amplified in two fragments with the primers oJKLiF-133/-134 and oJKLiF-135/-136. The resulting fragments were fused with an additional PCR using primers oJKLiF-133/-136 and transferred to Yeast MoClo entry vector [23]. TRP3 gene was PCR amplified with the primers oJKLiF-137/-138 and transferred to MoClo entry vector. Yeast MoClo method was used to construct integration cassettes for 
$T R P 2^{S 76 L}$ and TRP3. TRP2 $2^{S 76 L}$ cassette composed of the following building blocks: pYTK-002, -009, -051, -067, -074, -087, -089 and -093 resulting in LEU2 targeted integration cassette with $U R A 3$ selection marker and $T R P 2^{S 76 L}$ expressed under TDH3p. The corresponding composition of the TRP3 cassette was: pYTK-003, -011, $-052,-068,-075,-086,-089$ and -092 resulting in URA3 targeted integration cassette with $L E U 2$ selection marker and TRP3 expressed under PGK1p. Promoter switches for the genes ARO1, ARO2, GLN1 and CDC19 were carried out by using PCR amplified promoters flanked with homology arms as a donor DNA.

The expression cassette for the MtAAMT1 was constructed in the $S$. cerevisiae integrative plasmid targeting the HIS 3 locus. The plasmid was based on previously constructed pHIS3in-SpHIS5 and pGRE3in-kanMX plasmids [37]. The $k a n M X$ selection marker from the pGRE3inkanMX was cloned in place of the SpHIS5 marker in the pHIS3in-SpHIS5 between AscI and BglII restrictions sites to make the pHIS3in-kanMX plasmid. This plasmid was further modified by inserting DNA parts from plasmid pLEU2in-SES-A* [24]. Two DNA fragments from the pLEU2in-SES-A* were obtained by PCR with primers 8BS_mCh_GA_F and 8BS_mCh_GA_R and sTF_noPacI GA_F and sTF_noPacI_GA_R (Table 1), and these two fragment were assembled between $A s c I$ and EcoRI of the pHIS3in-kanMX by the Gibson assembly method (NEB). The resulting plasmid pHIS3in-kanMX_SES-A* was used for the final construction step, where the PCR product containing the S. cerevisiae codon-optimized MtAAMT1 coding region (MtAAMT1 from Medicago truncatula; NCBI Reference Sequence: XP_003604791.1; codon optimized and ordered as a synthetic gene (Genescript, USA) with suitable DNA flanks) was inserted between PacI and BamHI sites to form pHIS3in-kanMX_SES-MtAAMT1. The MtAAMT1 coding sequence was amplified by PCR using primers MtAAMT1_GA_F and MtAAMT1_GA_R (Table 1). The final expression cassette was confirmed by sequencing.

\section{S. cerevisiae genome editing}

For the CRISPR/Cas9 mediated genomic integrations, an approach based on a two plasmid system-Cas 9 and single-guide RNA (sgRNA) expressed from separate plasmids-was used similar as described previously [18]. The Cas9 expression vector was constructed from Yeast MoClo parts pYTK-002, -013, -036, -051, -067, -077, -081 and -083 resulting in a CEN/ARS plasmid with $\mathrm{KanR}$ selection marker and Cas 9 expressed under TEF1p. All the sgRNA plasmids were based on a backbone which was constructed from the Yeast MoClo parts pYTK002, -013, -051, -067, -078, -082 and -083 resulting in a $2 \mu$ plasmid with NatR selection marker. This backbone was PCR amplified with the primers oNNF-003/-004 and was co-transformed into $S$. cerevisiae together with a PCR amplified DNA fragment containing an expression cassette for sgRNAs (expressed between pSNR52 and $t S U P 4)$. The workflow of the CRISPR/Cas9 method is described in the Additional file 1: Fig. S1 and the oligos for construction of each sgRNA expression cassettes are listed in Table 1. All S. cerevisiae transformations were carried out by using the Gietz transformation method [38]. About $0.5-1 \mu \mathrm{g}$ of sgRNA plasmid backbone and expression cassette and 1-5 $\mu \mathrm{g}$ of donor DNA was used in the transformations.

\section{Chemical analyses}

The yeast liquid cultures were centrifuged and ANTH / Me-ANTH titers were analyzed from the supernatants. UPLC was carried out using ACQUITY UPLC BEH HSS T3 Column, $1.8 \mu \mathrm{m}, 2.1 \mathrm{~mm}$ X $100 \mathrm{~mm}$ (Waters) kept at $45^{\circ} \mathrm{C}$. An injection volume of $2 \mu \mathrm{l}$ was used. Flow rate of the mobile phase $\mathrm{A}(0.1 \%$ of formic acid in water $)$ and $\mathrm{B}$ $\left(0.1 \%\right.$ of formic acid in methanol) was $0.4 \mathrm{ml} \mathrm{min}^{-1}$. Following gradient program was used: $5.0 \%$ of B at $0.0 \mathrm{~min}$, $60.9 \%$ of B at $4.3 \mathrm{~min}, 97.1 \%$ of B at $5.6 \mathrm{~min}$, and $95.0 \%$ of $B$ at $6.0 \mathrm{~min}$. Equilibrium time of $2.0 \mathrm{~min}$ was used between runs.

Mass spectrometry was carried out using electrospray ionization (ESI) in positive polarity. The capillary voltage was $3.0 \mathrm{kV}$, cone voltage $30 \mathrm{kV}$, source temperature $150{ }^{\circ} \mathrm{C}$ and desolvation temperature $500{ }^{\circ} \mathrm{C}$. The cone and desolvation gas flow were set at $150 \mathrm{l} \mathrm{h}^{-1}$ (nitrogen) and $1000 \mathrm{l} \mathrm{h}^{-1}$ (nitrogen), respectively, collision gas was $0.15 \mathrm{ml} \mathrm{min}^{-1}$. The analysis was performed with ANTH and Me-ANTH as analytical standards for the identification and quantification of the products.

\section{Supplementary Information}

The online version contains supplementary material available at https://doi. org/10.1186/s12934-021-01532-3.

Additional file 1: Fig. S1 The workflow of the CRISPR/Cas9 method used in the strain construction. The S. cerevisiae strain was first transformed with the Cas9 expression vector and then with the linear fragments of the sgRNA expression cassette, the sgRNA expression plasmid backbone and the donor DNA. The sgRNA expression cassettes were produced by two $P C R$ reactions. The first PCR produces the target specific 20 nucleotide sequence and results in two fragments. The second PCR then amplifies the whole cassette fusing these fragments together. The sgRNA expression plasmid is formed by homologous recombination in vivo from the sgRNA expression cassette and the plasmid backbone. After each round of genomic integrations, the sgRNA expression plasmid is dropped from the cells by growing on non-selective medium which allows new round of modification to be carried out. Fig. S2 The expression cassette used for the MtAAMT1 expression in the S. cerevisiae strain engineered for ANTH production (strain 4). Fig. S3 Example of UPLC-MS analysis of ANTH and Me-ANTH. The top panel represents the base peak intensity (BPI) chromatograms of analytical standards, ANTH and Me-ANTH as detected by mass 
spectrometry. The lower panels show the same analysis of production of the compounds by the strain 4 and the strain 4 modified by the expression cassette for MTAAMT1.

\section{Abbreviations}

ANTH: Anthranilate; CCM: Cis,cis-muconic acid; CHO: Chorismate; DAHP: 3-Deoxy-D-arabino-heptulosonate-7-phosphate; DHQ: 3-Dehydroquinate; DHS: 3-Dehydroshikimate; E4P: Erythrose-4-phosphate; EPSP: 5-Enolpyruvylshikimate-3-phosphate; ESI: Electrospray ionization; F6P: Fructose-6-phosphate; G3P: Glyceraldehyde-3-phosphate; GLC: Glucose; Glu: Glutamate; GIn: Glutamine; Me-ANTH: Methyl anthranilate; MtAAMT1: Medicago truncatula Anthranilic acid methyltransferase 1; PEP: Phosphoenolpyruvate; Phe: Phenylalanine; PPP: Pentose phosphate pathway; PYR: Pyruvate; S3P: Shikimate3-phosphate; SA: Shikimate; SES: Synthetic expression system; Trp: Tryptophan; Tyr: Tyrosine; X5P: Xylulose-5-phosphate.

\section{Acknowledgements}

We thank Sami Holmström, Ulla Lahtinen, Natalia Mayorova and Heli Nygren at VTT Industrial Biotechnology for their assistance.

\section{Authors' contributions}

JK designed and carried out the experiments related to ANTH production. JK and MK analyzed the results and wrote the manuscript. DM designed and carried out the experiments related to Me-ANTH production, participated in analyzing the results and writing of the manuscript. HR and MT participated in designing the Me-ANTH production experiments, analyzing the results and writing of the manuscript. JJ participated in analysing the results, writing of the manuscript and supervision of the work. All authors read and approved the final manuscript.

\section{Funding}

This work was supported by The Novo Nordisk Foundation Grant NNF170C0025726.

\section{Availability of data and materials}

The datasets used and/or analysed during the current study are available from the corresponding author on reasonable request.

\section{Ethics approval and consent to participate} Not applicable.

\section{Consent for publication}

Not applicable.

\section{Competing interests}

The authors declare that they have no competing interests.

\section{Author details}

${ }^{1}$ VTTT Technical Research Centre of Finland Ltd, Espoo, Finland. ${ }^{2}$ Present Address: eniferBio Oy, Espoo, Finland.

Received: 31 August 2020 Accepted: 27 January 2021

Published online: 03 February 2021

\section{References}

1. Thompson B, Machas M, Nielsen DR. Creating pathways towards aromatic building blocks and fine chemicals. Curr Opin Biotechnol. 2015;36:1-7.

2. Lee J, Wendisch VF. Biotechnological production of aromatic compounds of the extended shikimate pathway from renewable biomass. J Biotechnol. 2017;257:211-21.

3. Noda S, Kondo A. Recent advances in microbial production of aromatic chemicals and derivatives. Trends Biotechnol. 2017;35:785-96.

4. Gottardi M, Reifenrath M, Boles E, Tripp J. Pathway engineering for the production of heterologous aromatic chemicals and their derivatives in Saccharomyces cerevisiae: bioconversion from glucose. FEMS Yeast Res. 2017. https://doi.org/10.1093/femsyr/fox035.
5. Kuepper J, Dickler J, Biggel M, Behnken S, Jäger G, Wierckx N, et al. Metabolic engineering of Pseudomonas putida KT2440 to produce anthranilate from glucose. Front Microbiol. 2015;6:1-9.

6. Wiklund P, Bergman J. The chemistry of anthranilic acid. Curr Org Synth. 2006:3:379-402.

7. Widhalm JR, Dudareva N. A familiar ring to it: biosynthesis of plant benzoic acids. Mol Plant. 2015;8:83-97.

8. Balderas-Hernández VE, Sabido-Ramos A, Silva P, Cabrera-Valladares N, Hernández-Chávez G, Báez-Viveros JL, et al. Metabolic engineering for improving anthranilate synthesis from glucose in Escherichia coli. Microb Cell Fact. 2009:8:1-12.

9. Luo ZW, Cho JS, Lee SY. Microbial production of methyl anthranilate, a grape flavor compound. Proc Natl Acad Sci USA. 2019;166:10749-56.

10. Sun X, Lin Y, Huang Q, Yuan Q, Yan Y. A novel muconic acid biosynthesis approach by shunting tryptophan biosynthesis via anthranilate. Appl Environ Microbiol. 2013;79:4024-30.

11. Hong KK, Nielsen J. Metabolic engineering of Saccharomyces cerevisiae: a key cell factory platform for future biorefineries. Cell Mol Life Sci. 2012;69:2671-90.

12. Jiang H, Morgan JA. Optimization of an in vivo plant P450 monooxygenase system in Saccharomyces cerevisiae. Biotechnol Bioeng. 2004:85:130-7.

13. Eudes A, Benites VT, Wang G, Baidoo EEK, Lee TS, Keasling JD, et al. Precursor-directed combinatorial biosynthesis of cinnamoyl, dihydrocinnamoyl, and benzoyl anthranilates in Saccharomyces cerevisiae. PLoS ONE. 2015;10:1-19.

14. Jäger G, Moussa A, Klaffl S, Hamedinger T, Kloeckner W, Behnken S. WO2017/102853 A1. 2017.

15. Duncan K, Edwardst RM, Coggins JR. The pentafunctional arom enzyme of Saccharomyces cerevisiae is a mosaic of monofunctional domains. Biochem J. 1987;246:375-86.

16. Blank LM, Kuepfer L, Sauer U. Large-scale 13C-flux analysis reveals mechanistic principles of metabolic network robustness to null mutations in yeast. Genome Biol. 2005;6:1-16.

17. Vaseghi S, Baumeister A, Rizzi M, Reuss M. In vivo dynamics of the pentose phosphate pathway in Saccharomyces cerevisiae. Metab Eng. 1999;1:128-40.

18. Kuivanen J, Holmström S, Lehtinen B, Penttilä M, Jäntti J. A highthroughput workflow for CRISPR/Cas9 mediated combinatorial promoter replacements and phenotype characterization in yeast. Biotechnol J. 2018;13:1700593

19. Jessop-Fabre MM, Jakočiūnas T, Stovicek V, Dai Z, Jensen MK, Keasling JD, et al. EasyClone-MarkerFree: A vector toolkit for marker-less integration of genes into Saccharomyces cerevisiae via CRISPR-Cas9. Biotechnol J. 2016;11:1110-7.

20. Hartmann M, Schneider T, Pfeil A, Heinrich G, Lipscomb WN, Braus G. Evolution of feedback-inhibited beta/alpha barrel isoenzymes by gene duplication and a single mutation. GBM Annu Fall Meet Halle. 2002;2003(100):862-7.

21. Graf R, Mehmann B, Braus GH. Analysis of feedback-resistant anthranilate synthases from Saccharomyces cerevisiae. J Bacteriol. 1993;175:1061-8.

22. Toyn JH, Gunyuzlu P, White WH, Thompson LA, Hollis GF. A counterselection for the tryptophan pathway in yeast: 5-fluoroanthranilic acid resistance. Yeast. 2000;16:553-60.

23. Lee ME, DeLoache WC, Cervantes B, Dueber JE. A highly characterized yeast toolkit for modular, multipart assembly. ACS Synth Biol. 2015;4:975-86

24. Rantasalo A, Landowski CP, Kuivanen J, Korppoo A, Reuter L, Koivistoinen $\mathrm{O}$, et al. A universal gene expression system for fungi. Nucleic Acids Res. 2018:46:e111.

25. Luttik MAH, Vuralhan Z, Suir E, Braus GH, Pronk JT, Daran JM. Alleviation of feedback inhibition in Saccharomyces cerevisiae aromatic amino acid biosynthesis: quantification of metabolic impact. Metab Eng 2008; 10:141-53.

26. Curran KA, Leavitt JM, Karim AS, Alper HS. Metabolic engineering of muconic acid production in Saccharomyces cerevisiae. Metab Eng. 2013;15:55-66

27. Gold ND, Gowen CM, Lussier FX, Cautha SC, Mahadevan R, Martin VJJ. Metabolic engineering of a tyrosine-overproducing yeast platform using targeted metabolomics. Microb Cell Fact. 2015;14:1-16. 
28. Wang J, Zhang R, Zhang Y, Yang Y, Lin Y, Yan Y. Developing a pyruvatedriven metabolic scenario for growth-coupled microbial production. Metab Eng. 2019;55:191-200.

29. Mao J, Liu Q, Song X, Wang H, Feng H, Xu H, et al. Combinatorial analysis of enzymatic bottlenecks of l-tyrosine pathway by p-coumaric acid production in Saccharomyces cerevisiae. Biotechnol Lett. 2017;39:977-82.

30. Solovjeva ON, Kochetov GA. Inhibition of transketolase by p-hydroxyphenylpyruvate. FEBS Lett. 1999;462:246-8.

31. Suástegui M, Yu Ng C, Chowdhury A, Sun W, Cao M, House E, et al. Multilevel engineering of the upstream module of aromatic amino acid biosynthesis in Saccharomyces cerevisiae for high production of polymer and drug precursors. Metab Eng. 2017;42:134-44.

32. Dodge TC, Gerstner JM. Optimization of the glucose feed rate profile for the production of tryptophan from recombinant E. coli. J Chem Technol Biotechnol. 2002;77:1238-45.

33. Boles E, De Jong-Gubbels P, Pronk JT. Identification and characterization of MAE1, the Saccharomyces cerevisiae structural gene encoding mitochondrial malic enzyme. J Bacteriol. 1998;180:2875-82.

34. Pollier J, De Geyter N, Moses T, Boachon B, Franco-Zorrilla JM, Bai Y, et al. The MYB transcription factor emission of methyl anthranilate 1 stimulates emission of methyl anthranilate from Medicago truncatula hairy roots. Plant J. 2019;99:637-54.

35. Verduyn C, Postma E, Scheffers W, Van Dijken J. Effect of benzoic acid on metabolic fluxes in yeasts: a continuous-culture study on the regulation of respiration and alcoholic fermentation. Yeast. 1992;8:501-5017.

36. Verho R, Putkonen M, Londesborough J, Penttilä M, Richard P. A novel $\mathrm{NADH}$-linked $\mathrm{L}$-xylulose reductase in the $\mathrm{L}$-arabinose catabolic pathway of yeast. J Biol Chem. 2004;279:14746-51.

37. Rantasalo A, Kuivanen J, Penttilä M, Jäntti J, Mojzita D. Synthetic toolkit for complex genetic circuit engineering in Saccharomyces cerevisiae. ACS Synth Biol. 2018;7:1573-87.

38. Gietz R, Schiestl R. High-efficiency yeast transformation using the LiAc/SS carrier DNA/PEG method. Nat Protoc. 2007;2:31-4.

\section{Publisher's Note}

Springer Nature remains neutral with regard to jurisdictional claims in published maps and institutional affiliations.
Ready to submit your research? Choose BMC and benefit from:

- fast, convenient online submission

- thorough peer review by experienced researchers in your field

- rapid publication on acceptance

- support for research data, including large and complex data types

- gold Open Access which fosters wider collaboration and increased citations

- maximum visibility for your research: over $100 \mathrm{M}$ website views per year

At BMC, research is always in progress.

Learn more biomedcentral.com/submissions 